\section{Ziprasidone Associated Neuroleptic Malignant Syndrome}

Jeffrey D. Bell, M.D. ${ }^{1}$, Chancen Hall, D.O. ${ }^{2}$, Justin Sandall, D.O. ${ }^{2}$

${ }^{1}$ Beaver Medical Group, Redland, CA

${ }^{2}$ University of Kansas School of Medicine-Wichita, Wichita, KS

Department of Anesthesiology

Received Oct. 7, 2019; Accepted for publication Dec. 21, 2020; Published online March 19, 2021 https: doi.org 10.17161 kjm.voll411970

\section{INTRODUCTION}

Neuroleptic malignant syndrome (NMS) is a severe and potentially lethal disorder due to an adverse reaction to dopamine receptor antagonism or the rapid withdrawal of dopaminergic medications. ${ }^{1}$ While incidence is estimated to be $0.02 \%^{1}$ generally and $0.2 \%^{2}$ among neuroleptic users, NMS continues to be an unpredictable and potentially life-threatening condition of which case reports continue to be a main source of information for clinicians. ${ }^{3}$

Despite the advent of second-generation antipsychotics (SGA), NMS continues to be a rare yet severe adverse reaction to neuroleptics. ${ }^{4}$ It is seen most often with abrupt cessation, decrease, or increase in dopaminergic dose. ${ }^{5}$ A recent systematic literature review conducted by Murri et al. ${ }^{5}$ found only 186 individual cases meeting their criteria for SGA-NMS, only six of which were caused by ziprasidone. Our patient met the Diagnostic and Statistical Manual of Mental Disorders, Revised Fifth Edition, ${ }^{6}$ (DSM-V) diagnostic criteria for NMS as she presented with hyperthermia, profuse diaphoresis, generalized muscle rigidity, elevated creatinine phosphokinase, delirium, and autonomic instability. All were associated with exposure to a dopamine receptor antagonist, in this case, ziprasidone. While most patients fully recover and mortality rates have been decreasing due to early recognition and treatment, fatality rates are estimated between $5-10 \% .^{1-3}$ This case demonstrated how NMS can present as a mimic of sepsis or serotonin syndrome.

\section{CASE REPORT}

A 60-year-old female nursing home patient with bipolar affective disorder, hypertension, hypothyroidism, and paraplegia demonstrated increasing confusion requiring admission to a senior behavioral facility. Over a period of approximately a week and a half, she became unable to communicate and developed tachycardia, tachypnea, flushing, diaphoresis, and fevers greater than $38^{\circ} \mathrm{C}$. She was transferred to the Neurologic Critical Care Unit (NCCU) for further evaluation and treatment for possible sepsis.

Upon arrival to the NCCU, she was encephalopathic, tachypneic, tachycardic, and hypoxemic. She had generalized rigidity, although not lead pipe, of the trunk and extremities and $2+/ 4$ hyperreflexia without clonus which was difficult to evaluate with her baseline lower extremity paraplegia. Initial laboratory analysis revealed leukocytosis, acute kidney injury, elevated serum creatinine phosphokinase of $444 \mathrm{U} / \mathrm{L}$, procalcitonin elevated at $0.27 \mathrm{ng} / \mathrm{mL}$, and C-reactive protein levels which were within normal limits. While her initial blood pressure was normal, she had pronounced fluctuations with episodes of hypotension and hypertension.

Given these initial findings, the differential diagnosis included sepsis,
KANSAS JOURNAL of MEDICINE

NMS, serotonin syndrome (SS), non-convulsive status epilepticus, or other central nervous system (CNS) pathology. Blood, urine, and sputum cultures were obtained, and she was considered for a lumbar puncture to evaluate for meningitis. In addition, medical records were obtained from the outside facility and showed she had been receiving increasing doses of ziprasidone along with haloperidol, lithium, duloxetine, oxybutynin, and baclofen

Once this information was received and in consultation with our neurology and psychiatry colleagues, the patient was treated for a presumptive diagnosis of NMS with IV bromocriptine $10 \mathrm{mg}$ every six hours and a one-time dose of IV dantrolene $2.5 \mathrm{mg} / \mathrm{kg}$, discontinuation of possible causative agents, a cooling blanket and antipyretics, and IV fluids.

SS, which presents with dysautonomia and hyperreflexia in the setting of serotonergic medications, was considered for this patient, but the escalating doses of ziprasidone were the most influential factor compared to her unaltered use of duloxetine and lithium. Also, our cautious decision to administer bromocriptine was an SS challenge as bromocriptine likely would have exacerbated her condition if it had been serotonin related. ${ }^{7}$ The neurology consultant further recommended scheduling dantrolene at $2.5 \mathrm{mg}$ every six hours. Electroencephalography showed generalized slowing consistent with encephalopathy, but no seizure activity and magnetic resonance imaging of the brain with and without contrast showed no acute finding.

By hospital day three, the patient was afebrile with improved leukocytosis without antibiotics thus no lumbar puncture was performed. By day four, autonomic dysregulation had resolved with cessation of labile blood pressures. Supplemental oxygen requirements ceased, and she became more awake and less rigid in the extremities. However, she was unable to participate in a meaningful interview with psychiatry and dantrolene was increased to $100 \mathrm{mg}$ TID due to persistent rigidity. The following day she remained stable and was transferred out of the NCCU.

\section{DISCUSSION}

NMS is thought to be due primarily to neuroleptic blockade at the dopamine receptor resulting in reduced CNS dopaminergic tone and dysregulation of autonomic nervous system activity. ${ }^{5}$ Nonneuroleptic agents with antidopaminergic activity also have been associated with NMS such as metoclopramide, promethazine, tetrabenazine, droperidol, diatrizoate, and amoxapine. ${ }^{1}$ However, dopaminergic activity did not explain the pathogenic mechanism of NMS completely, because it also was induced by withdrawal of antipsychotics or by SGAs that have reduced action at dopamine receptors. There is increasing investigation into other receptors (i.e., serotonergic, adrenergic, and cholinergic) that may play an important role. ${ }^{5}$ There is also at least one case report of NMS-like syndrome due to baclofen withdrawal. ${ }^{8}$ Our patient's medication regimen gives credence to this theory as she not only had a recent increase in her ziprasidone to a maximum dose, but also was on haloperidol as needed for agitation, a typical antipsychotic, lithium, 
KANSAS JOURNAL of MEDICINE NEUROLEPTIC MALIGNANT SYNDROME continued.

which has anti-dopaminergic activity ${ }^{9}$, duloxetine, a serotonin reuptake inhibitor, and oxybutynin, which has anticholinergic activity. Furthermore, she improved clinically with the cessation of all these medications along with continuing baclofen.

The treatment of NMS is individualized and based on the clinical presentation. ${ }^{1}$ The first and most common step is cessation of the suspected offending agents. However, if the syndrome occurs after abrupt withdrawal of a dopaminergic medication, then the medication must be reinstituted. The second step involves supportive care typically consisting of aggressive hydration especially in cases of rhabdomyolysis, treatment of hyperthermia, and correction of metabolic abnormalities.

NMS patients are at increased risk for renal failure, disseminated intravascular coagulation (DIC) secondary to rhabdomyolysis, deep venous thrombosis, and pulmonary embolism from dehydration and immobilization, as well as aspiration pneumonia due to difficulty swallowing combined with altered mental status. ${ }^{1}$ Other medical complications include cardiopulmonary failure, seizures, arrhythmias, myocardial infarction, and sepsis. As such, many cases require intensive care monitoring and support.

In severe cases, such as ours, patients often are treated with empiric bromocriptine mesylate and dantrolene sodium. ${ }^{10}$ Bromocriptine mesylate is a dopamine agonist used to reverse the hypodopaminergic state and is administered orally or via a nasogastric tube starting with $2.5 \mathrm{mg}$ two to three times daily and increasing the dose by $2.5 \mathrm{mg}$ every 25 hours until a response or until reaching a maximum dose of $45 \mathrm{mg} / \mathrm{d} .{ }^{1}$ Bromocriptine generally is continued for at least 10 days in NMS related to oral antipsychotics and two to three weeks for depot neuroleptics.

Dantrolene sodium is a muscle relaxant that works by inhibiting calcium release from the sarcoplasmic reticulum and is administered intravenously starting with a loading dose of $1-2.5 \mathrm{mg} / \mathrm{kg}$ followed by $\mathrm{l}$ $\mathrm{mg} / \mathrm{kg}$ every six hours up to a maximum dose of $10 \mathrm{mg} / \mathrm{kg} / \mathrm{day} .{ }^{1}$ Regular monitoring of liver function is recommended with dantrolene due to its risk for hepatotoxicity. However, while often noted to aid some patients, Reulbach et al. ${ }^{11}$ showed that there was no evidence supporting the use of dantrolene as a treatment of NMS. This reiterated the need to understand the etiology of NMS to find a better treatment.

\section{CONCLUSIONS}

Second generation antipsychotics are the most commonly prescribed antipsychotics, but our knowledge of SGA-NMS continues to be limited due its sporadic and rare nature. ${ }^{1}$ As such, case reports continue to be a main source of information for clinicians. ${ }^{3}$ Many of the signs of NMS are non-specific and qualify the patients for the systemic inflammatory response syndrome (SIRS). Our patient was transferred from an outside behavioral health facility due to worsening encephalopathy and SIRS thought to be secondary to sepsis. It was only after arrival to the NCCU and a thorough physical exam and review of outside records that NMS became the primary etiology in the differential diagnosis. NMS is a rarely described phenomenon, particularly with newer agents such as ziprasidone.

To our knowledge, this is one of only a few case reports of NMS presenting as a sepsis mimic. ${ }^{3}$ Our case highlighted the need for increased study into the potential role of multiple receptors in NMS as well as the need for a high index of suspicion for those patients who present with SIRS, encephalopathy, and rigidity while on psychotropic medications.

\section{REFERENCES}

${ }^{1}$ Berman BD. Neuroleptic malignant syndrome: A review for neurohospitalists. Neurohospitalist 2011; 1(1):41-47. PMID: 23983836.

${ }^{2}$ Modi S, Dharaiya D, Schultz L, Varelas P. Neuroleptic malignant syndrome: Complications, outcomes, and mortality. Neurocrit Care 2016;24(1):97-103. PMID: 26223336

${ }^{3}$ Patel K, Lilly B, Ajayi O, Melvin K. A case of neuroleptic malignant syndrome in a profoundly intellectually disabled patient with successful reintroduction of antipsychotic therapy with quetiapine. Case Rep Psychiatry 2018; 2018:7045106. PMID: 30112240.

${ }^{4}$ Stübner S, Rustenbeck E, Grohmann R, et al. Severe and uncommon involuntary movement disorders due to psychotropic drugs. Pharmacopsychiatry 2004; 37(1):S54-S64. PMID: 15052515.

${ }^{5}$ Belvederi Murri M, Guaglianone A, Bugliani M, et al. Second-generation antipsychotics and neuroleptic malignant syndrome: Systematic review and case report analysis. Drugs R D 2015; 15(1):45-62. PMID: 25578944.

${ }^{6}$ American Psychiatric Association. Diagnostic and Statistical Manual of Mental Disorders, Revised Fifth Edition. Washington, DC: American Psychiatric Association, 2013.

7 Dosi R, Ambaliya A, Joshi H, Patell R. Serotonin syndrome versus neuroleptic malignant syndrome: A challenging clinical quandary. BMJ Case Rep 2014; 2014:bcr2014204154. PMID: 24957740.

8 Turner MR, Gainsborough N. Neuroleptic malignant-like syndrome after abrupt withdrawal of baclofen. J Psychopharmacol 2001; 15(1):61-63. PMID: 11277611.

9 Beaulieu JM, Sotnikova TD, Yao WD, et al. Lithium antagonizes dopamine-dependent behaviors mediated by an AKT/glycogen synthase kinase 3 signaling cascade. Proc Natl Acad Sci USA 2004; 101(14):5099-5104. PMID: 15044694

${ }_{10}$ Sakkas P, Davis JM, Janicak PG, Wang ZY. Drug treatment of the neuroleptic malignant syndrome. Psychopharmacol Bull 1991; 27(3):381-384. PMID: 1685592.

${ }^{11}$ Reulbach U, Dütsch C, Biermann T, et al. Managing an effective treatment for neuroleptic malignant syndrome. Crit Care 2007; 11(1): R4. PMID: 17222339.

Keywords: ziprasidone, neuroleptic malignant syndrome, antipsychotic agents 